\title{
Haptic Feedback Control in Medical Robots through Fractional Viscoelastic Tissue Model
}

\author{
Yo Kobayashi, Pedro Moreira, Chao Liu, Philippe Poignet, Nabil Zemiti, Masakatsu G. Fujie
}

\begin{abstract}
In this paper, we discuss the design of an adaptive control system for robot-assisted surgery with haptic feedback. Through a haptic device, the surgeon teleoperates the medical instrument in free space, fixed on a remote robot or in contact. In free space, the surgeon feels the motion of the robot. In the present paper, we evaluated the performance of the controller on viscoelastic tissue, modeled by a fractional derivative equation. In addition, we propose a novel controller using an integer formalization process that is suitable for these tissue properties. The simulation results suggested that performance, in terms of force control and telepresence, became poorer when the conventional controller, which was designed for elastic target object, was applied to the viscoelastic tissues. In contrast, the results suggested that our proposed controller maintained its performance on the viscoelastic tissues.
\end{abstract}

\section{INTRODUCTION}

\section{A. Haptic feedback}

The minimally invasive surgery (MIS) has become widespread in the field of medical intervention like surgery, biopsy, percutaneous therapy. The surgeon, with the use of visual feedback, manipulates and guides a dedicated surgical instrument that enters in the human body through a penetration point. These medical techniques result in less operative trauma and reduce the incidence of post-surgical complications for the patient. The use of a robotic teleoperation system can greatly enhance surgeon dexterity, skills and security. In the field of teleoperated medical robotic systems, the remote robot, in which the surgical instrument (or needle) is mounted, is tele-controlled by the surgeon to achieve the medical gestures. Aside from visual feedback, the surgeon has no other feedback information to detect contact with the tissue. For example, the Da VinciTm system from Intuitive Surgical is the state-of-the-art robotic system for MIS, in which surgeon hand movements are scaled, filtered and sent to the robotic end-effector. This setup has been used in many MIS, such as cardiac, urology and other abdomen surgeries, without haptic feedback.

Since surgeons largely rely on their haptic feeling to detect and characterize contact with tissues, organs or flesh, it is

Manuscript received March 26, 2011. This work was supported in part by Global COE (Centers of Excellence) Program "Global Robot Academia," Waseda University, Tokyo, Japan; and in part by Institutional Program for Young Researcher Overseas Visit from Japan Society for the Promotion of Science (JSPS).

Y. Kobayashi is with Faculty of Science and Engineering, Waseda University, Japan, (59-309, 3-4-1, Ohkubo Shinjuku, Tokyo, Japan, phone: +81-3-5286-3412; fax: +81-3-5291 -8269; e-mail: you-k@fuji.waseda.jp).

P. Moreira, C. Liu, Z. Nabil, P. Poignet are with LIRMM-UMR CNRS University of Montpellier II, F-34095 Montpellier cedex 5, France

M.G. Fujie is with Graduate School of Science and Engineering, Waseda University, Japan and Faculty of Science and Engineering, Waseda University, Japan very important that the teleoperation system can feedback reliably and faithfully the texture of the remote environment to the human operator. Therefore, telepresence is the major goal in teleoperation system's design. Guaranteed stability in contact with soft and stiff objects is an important issue.

\section{B. Problems}

Aside from technological problems linked to the non-existence of sterile force sensor, nowadays, surgical robotized systems allowing teleoperation, do not include force feedback to avoid instability problems. Robotized MIS does not include force feedback in the main control loop to avoid instability problems. Being able to operate a remote robot and touch an organ, a bone, another instrument or a rib for cardiothoracic surgery, requires advanced control techniques. Guaranteed stability in contact with stiff objects is an important milestone (e.g. touching another instrument or a rib for cardiothoracic surgery) which demands advanced control techniques, being a major obstacle for practical applications.

\section{Related works}

Force feedback to the surgeon will be essential for safe surgery. Then, many approaches have been proposed to investigate the issue of stability and transparency in time-delayed teleoperation systems [1]-[3]. In the field of medical robotic systems, the master and slave station are considered in the same operating room and the time delay is considered to be small. In [4], based on the transparency concept in bilateral teleoperation, a new fidelity measure is proposed. Using a one degree of freedom (DOF) system, only soft objects are considered without free space/contact transitions. For surgical tasks which involve surgical needle gestures, many efforts have been reported to help the surgeon perform the medical intervention [5]-[6]. The proposed strategies consider the design of robotic and medical imaging systems to help the surgeon in the needle guidance task. A teleoperated architecture is proposed without considering the haptic feedback in [7]. To give haptic clues to the physician during the needle insertion, an additional statistical technique is used to detect the puncture time and the transition between tissues in [8]. In [9], the stability of the teleoperation scheme is achieved using heuristic techniques to estimate the environment parameter. This approach gives good results but it depends on the local force controller parameters and is not easy to tune.

\section{Objectives}

In this paper, we propose an adaptive haptic feedback control design for a medical robotics teleoperation system. The force feedback controller uses feedback linearization and stochastic state observer design. This incorporates a 
systematic control design for robotized MIS, using operational and null space techniques [10] linked to Active Observers (AOBs) [11]. It extends previous work [12] by the inclusion of haptic feedback and eliminating gradient-based functions from the control design. To realize a robust and efficient teleoperation system, the controller uses information on environment structure. The estimated environment parameters are used to generate the robot trajectory. Based on previous analyses, teleoperation scheme performances were improved for medical application [13]-[16].

In the previous studies [13]-[16], environment structure was assumed to be elastic and modeled as a spring. However, in general, tissue modeling is complex because tissue has elastic and viscous characteristics that together are called viscoelasticity. It is well known that biomaterial such as soft tissue have special dynamic properties. In fact, researchers (including our group) have reported [17]-[19] that the viscoelasticity of a biomaterial has different properties from conventional serial and/or parallel arrangements of springs (elasticity) and dashpots (viscosity) considered in models such as Voight model. These studies suggest that a "springpot", which is based on fractional calculus, accurately represents the viscoelastic properties of biomaterials, whereas the conventional viscoelastic model does not (Fractional calculus is a branch of mathematical analysis concerned with taking real or complex number powers of differential operators).

Our study objective is to investigate the performance of the controller previously proposed [13]-[16] when applied to the case of viscoelastic tissue now, which is modeled by a fractional derivative equation. In addition, we propose a novel controller that is suitable for tissue with these properties. We present simulations on the performance of the controller with one DOF robots. Experiments are proposed as the next step in this research.

The remainder of the present paper is organized as follows. Section II provides an overview of the teleoperation scheme previously proposed [13]-[16]. The simulation investigation for the viscoelastic model, which focuses on time-domain analysis of force controller and frequency domain analysis of telepresence, is addressed in Section III. Conclusions are presented in Section IV.

\section{HAPTIC FORCE CONTROLLER}

This section introduces the overview of the haptic controller in previous paper [13]-[16] which was designed for elastic target object. Novel controller that is suitable for viscoelastic tissue is presented in following section III. A picture of the teleoperation scheme proposed in [13]-[16] is represented in Fig.1. A decoupled force controller is described in the base frame by 3D (three dimensional) Cartesian position/force vectors, associated to the slave robot. The system transfer function $G(s)$ embeds operational space and feedback linearization techniques. The position of master $X_{h p}$ scaled by $\beta_{p}$ is compared to the end-effector position of slave $X_{s}$, generating a desired force $F_{d}$ through the virtual coupling $K_{v}$. The measured force $y_{k}=F_{m}$ is computed from the force sensor and projected into the base frame. It tracks

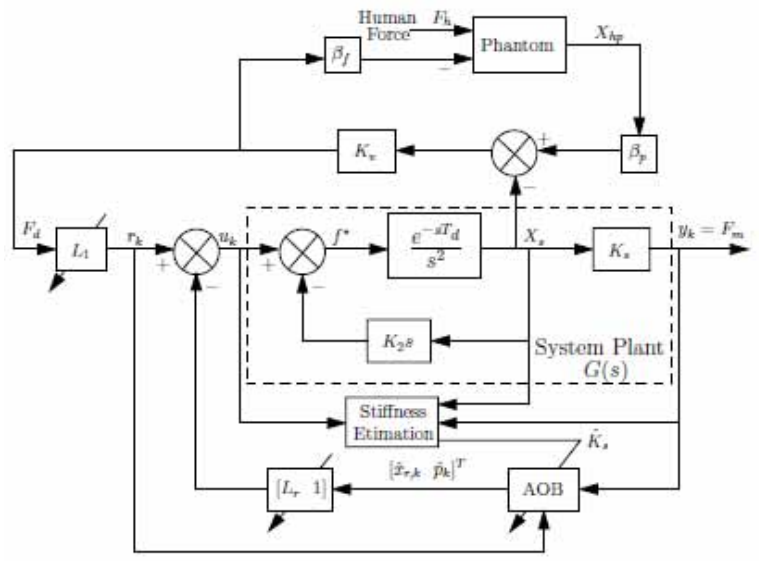

Fig. 1: Teleoperation control scheme with AOBs for each Cartesian dimension. The master station, which includes the human and phantom, generates the 3D Cartesian force $F_{d}$ through the virtual coupling $K_{v} . B_{p}$ scales the phantom position $\mathrm{X}_{\mathrm{hp}}$, and $\beta_{\mathrm{f}}$ scales back $\mathrm{F}_{\mathrm{d}}$ to the master station. $\mathrm{G}(\mathrm{s})$ has a damping term $\mathrm{K}_{2}$, and is controlled by AOB estimates $\left[\mathrm{x}_{\mathrm{r}, \mathrm{k}}, \mathrm{pk}\right]^{\mathrm{T}}$ through the state feedback gain $\left[\mathrm{L}_{\mathrm{r}} 1\right] . \mathrm{K}_{\mathrm{s}}$ is the system stiffness and $\mathrm{L}_{1}$ is the first element of $\mathrm{L}_{\mathrm{r}}$. The environment stiffness is estimated $\left(\mathrm{K}_{\mathrm{e} s}\right)$ and used to adapt the force controller.

the reference $F_{d}$ with desired dynamics through the state observer, even if the system stiffness $K_{s}$ changes. The human arm perceives $F_{d}$ scaled by $\beta_{f}$, which anticipates the real force. The estimated value $K_{e s, c}$ at time k is used to adapt the haptic force controller. The main advantage of such a position-position control scheme is that the human can feel position errors in free-space, due to motion, robot collision or human-robot interaction. Further details including comparisons between different teleoperation schemes are presented in [13]-[16].

\section{A. Manipulator Dynamics}

Using the operational space formulation, the dynamic model of the robot is given by

$$
M \ddot{q}+v(q, \dot{q})+g(q)=\tau
$$

where $M, V\left(q, q^{\circ}\right)$ and $g(q)$ are respectively the mass matrix, the Coriolis and centripetal force vector and the gravity term written in Cartesian coordinates. $F_{m}$ denotes the measured contact force at the end-effector of the manipulator. $F_{c}$ denotes the commanded force. For the desired Cartesian-decoupled system

$$
\ddot{X}_{s}=f^{*}
$$

in (1), $F_{c}$ should be

$$
F_{c}=F_{m}+V_{x}(q, \dot{q})+\hat{g}_{x}(q)+\Lambda_{x} f^{*}
$$

Introducing $k_{2}, T_{d}$ and $K_{s}$, the plant transfer function is given by (since $T_{d}$ is small)

$$
G(s)=\frac{K_{s} e^{-s T_{d}}}{s\left(s+k_{2} e^{-s T_{d}}\right)} \approx \frac{K_{s} e^{-s T_{d}}}{s\left(s+k_{2}\right)}
$$

Using the nominal value $K_{s}$ (for the desired plant), the equivalent time representation is

$$
\ddot{y}(t)+K_{2} \dot{y}(t)=K_{s, n u}\left(t-T_{d}\right)
$$

where $y(t)$ is the plant output (Cartesian force at the robot end-effector), and $u$ is the plant input (force). Defining the 
state variables $x_{l}(t)=y(t)$ and $\mathrm{x}_{2}(t)=y(t)$ and discretizing (5) with sampling time $\mathrm{h}$, the equivalent discrete time system is:

$$
\left\{\begin{array}{c}
x_{r, k}=\Phi_{r} x_{r, k-1}+\Gamma_{r} u_{k-1} \\
y_{k}=C_{r} x_{r, k}
\end{array}\right.
$$

with

$$
x_{r, k}=\left[\begin{array}{lllll}
x_{k} & u_{k-d} & \cdots & u_{k-2} & u_{k-1}
\end{array}\right]
$$

and $y_{k}=F_{m}$ is the measured force. Further details about the computation of the matrices $\Phi_{r}, \Gamma_{r}$ and $C_{r}$ are presented in [13].

\section{B. Force Controller Design}

To perform model reference adaptive control, the active observer AOB reformulates the Kalman filter based on a desired closed loop behavior (reference model) and on an extra state to enclose an equivalent disturbance referred to the system input [11]. These disturbances are due to unmodeled terms including higher order dynamics, parameter mismatches and unknown disturbances. An active state $p_{k}$ (extra state) describes these disturbances. Its estimate $p_{e k}$ performs the compensation action in the input through the state feedback (Fig. 1). Details of the AOB design can be seen in [13]. The state feedback gain $L_{r}$ has been tuned to limit overshoots/undershoots force response. It can be computed to achieve a critically damped system $(\zeta=1)$. The closed loop time constant $\tau_{c}$ (in our setup, $\tau_{c}=75[\mathrm{~ms}]$ ) should be small enough to enable the task execution with comfortable performance. The equivalent closed loop transfer function $G_{c l}(s)$ when the robot is in contact is

$$
G_{c l}=\frac{1}{\left(1+\tau_{c} s\right)^{2}} e^{-s T_{d}}=\frac{F_{m}}{F_{d}}
$$

\section{Teleoperation scheme}

Fig. 2 also illustrates the proposed teleoperation scheme. $G_{h p}$ is the master device (human and haptic device) transfer function. Considering a light haptic device, it can be represented by a mass-damper-spring system [20].

$$
G_{h p}=\frac{1}{M_{h p} s^{2}+C_{h p} s+K_{h p}}
$$

where $M_{h p}=0.8[\mathrm{Kg}], C_{h p}=100[\mathrm{Kg} / \mathrm{s}]$ and $K_{h p}=300[\mathrm{~N} / \mathrm{m}]$ are respectively the mass, damping and stiffness coefficients of the master station. The input of the master and slave is the desired force $F_{d}$. The force controller commands the slave station to track $F_{d}$ with a desired dynamic.

Telepresence is achieved if force tracking is achieved and if $K_{e s, c}$ represents the real value of the environment stiffness. The telepresence of the proposed teleoperation scheme can be analyzed by investigating the transmitted impedance $Z_{t}$ felt by the human. As shown in Fig. 1, the closed loop dynamic of the master operator is

$$
\frac{F_{h}}{X_{h p}}=G_{h p}+\frac{K_{v} \beta_{f} \beta_{p}}{1+G_{c l} K_{v} K_{s}}=Z_{t}
$$

For low frequencies, the transmitted impedance becomes

$$
Z_{t}=K_{h p}+\frac{K_{v} \beta_{f} \beta_{p}}{K_{v}+K_{s}} K_{s}
$$

From (11), a desired telepresence parameter $Z_{d t}$ can be defined by

$$
Z_{t}^{d}=K_{h p}+\alpha^{d} K_{s}
$$

The operator feels his hand, the phantom arm and the environment parameter $K_{s}$ scaled by $\beta_{d}$. Ideally, the teleoperation system should be completely transparent, so that the operator feels as if he is directly interacting with the remote task (and hence $\beta_{d}=1$ ).

\section{SimULATION INVESTIGATION}

This section describes the simulation investigation of the performance of the conventional and proposed controller applied to viscoelastic tissue.

\section{A. Tissue model}

We have previously studied biomechanical modeling of the viscoelastic properties of liver [17][18], breast [21] and muscle [22]. We modeled viscoelastic tissue properties using a single fractional derivative equation (13).

$$
K_{s} \frac{d^{r} X_{s}}{d t^{r}}=F_{m}
$$

where $r$ is the order of the derivative.

We set the value of $r$ as 0.125 based on the findings of a previous experiment [22].

\section{B. Controller using an integer formalization process}

The proposed controller suitable for viscoelastic tissue properties uses an "integer formalization process". The block diagram of the proposed controller is shown in Fig. 2. The controller is based on the conventional controller previously proposed [13]-[16] but includes the integer formalization process. No other change is needed in the structure of the controller. We added this fractional integration and derivative process (green block in Fig.2) to compensate for the effect of

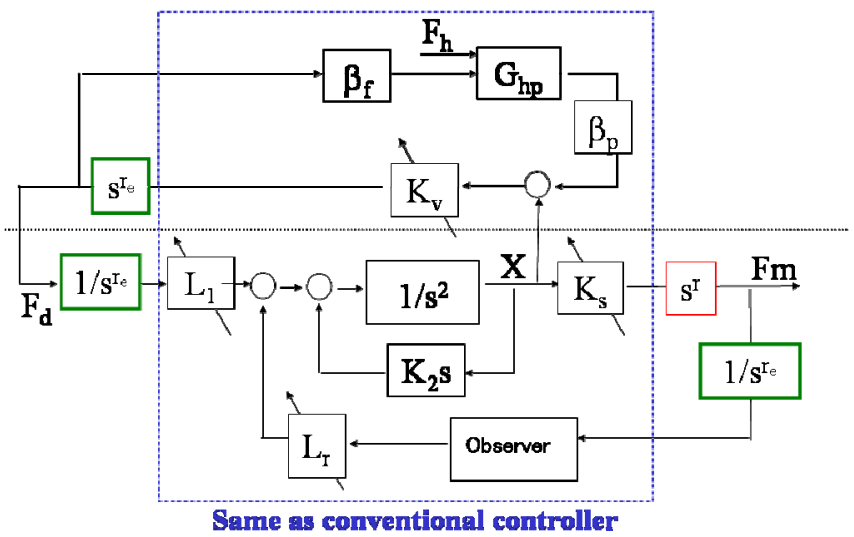

Fig. 2. Controller using an integer formalization process. Green blocks represent the additional fractional integration and derivative process. This process is provided to compensate for the effect of the viscoelasticity of the tissue (red block)

Table I. Parameters

\begin{tabular}{|c|c|c|c|}
\hline$K_{2}[\mathrm{Ns} / \mathrm{m}]$ & $\beta_{p}$ & $\beta_{f}$ & $\tau_{c}[\mathrm{~s}]$ \\
\hline 10 & 2.0 & 0.1 & 0.075 \\
\hline
\end{tabular}


the viscoelasticity of the tissue (red block in Fig.2). The blocks surrounded by the dotted line are the same as those of the conventional controller. The advantage of the integer formalization process is that we can use the same method and obtain the same performance as the conventional controller when the measured and desired virtual external forces $F_{m, v}$ and $F_{d, v}$ are used by the conventional controller. The model error between the derivative order of real tissue $r$ and the estimated value $r_{e}$ could decrease controller performance. Therefore, we investigated the robustness of the results on the performance of proposed controller to the model error.

\section{Time-domain analysis of force controller}

We simulated the step response of force control to evaluate the performance of the conventional and proposed controller. Time series data of the force $F_{m}$ was corrected when the desired force $F_{d}$ was 1.0 at constant (ref. the bottom part of Fig. 2). We also carried out simulations with different $r$ values for the surrounding tissues $(0.1,0.125,0.15)$ to evaluate the robustness of the results with respect to the variation in the parameter. The simulations were conducted with different estimated stiffness parameter $K_{s, e}=\left(1.0 * K_{s}\right.$, $2.0 * K_{s}$ ) of surrounding tissues.

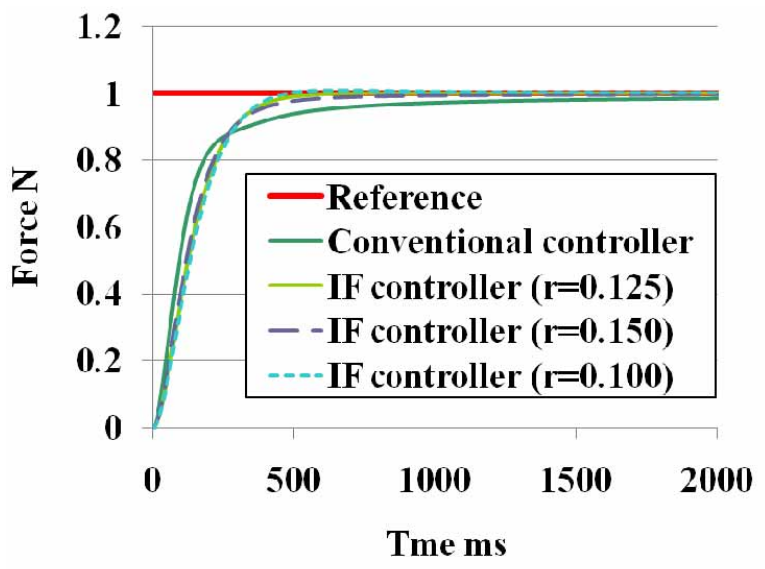

Fig. 3. Simulation results with correct estimated stiffness $\left(K_{s, e}=K_{s}\right)$

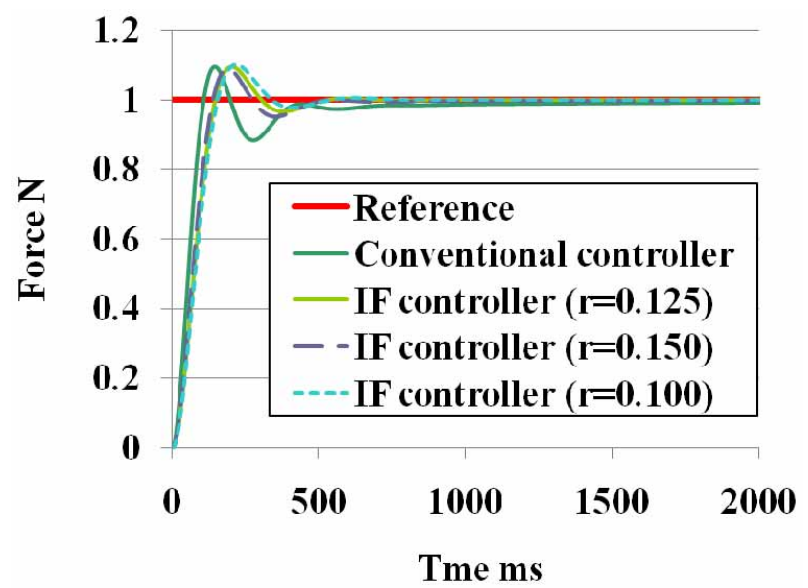

Fig. 4. Simulation results with higher estimated stiffness than that of Fig. 3 $\left(K_{s, e}=2.0 * K s\right)$
Figures 3 and 4 show the simulation results of the force $F_{m}$. Figure 3 presents the simulation results with the correct estimated stiffness $\left(K_{s, e}=K_{s}\right)$. The results in Fig. 3 show that the integer formalization controller provided the same results as those of a reference model (8). In contrast, the convergence of the force response was slow when using the conventional controller.

Figure 4 shows the simulation results with higher estimated stiffness than that of Fig. 3, $K_{s, e}=2.0 * K_{s}$. The results show that the force of both controllers overshot the asymptote. However, the overshoot of the integer formalization controller was smaller than that of the conventional controller. In addition, these simulation results (Figs 3 and 4) also suggested that the results using the alternative derivative parameters $r$ (equal successively to 0.1 and 0.15 ) only slightly differed from those using the correct derivative parameter $(r=0.125)$. Thus, the controller made only a small error if there was any model error. The force responses show that the transmitted was improved by using the integer formalization controller.

\section{Frequency-domain analysis of telepresence}

The telepresence of the teleoperation scheme can be assessed by the transmitted and desired impedance frequency-response. From equation (10), the impedance of the conventional controller with the considered phantom device is:

$$
Z_{t}=\frac{F_{h}}{X_{h p}}=G_{h p}^{-1}+K_{s} s^{r} \frac{K_{v} \beta_{s} \beta_{p}}{K_{v}+K_{s} s^{r}}
$$

The impedance of the controller with integer formalization:

$$
Z_{t}=\frac{F_{h}}{X_{h p}}=G_{h p}^{-1}+K_{s} s^{r} \frac{K_{v} s^{r_{e}} \beta_{s} \beta_{p}}{K_{v} s^{r_{e}}+K_{s} s^{r}}
$$

From (12) and (13), a desired telepresence parameter $Z_{t}$ can be defined by

$$
Z_{t}^{d}=\frac{F_{h}}{X_{h p}}=K_{h p}+\alpha^{d} K_{s} s^{r}
$$

The transmitted impedance $Z_{t}$ was computed with a fixed $K_{v}$ and $K_{s}=1000[\mathrm{~N} / \mathrm{m}]$. The other parameters are shown in Table 1. We also carried out the simulation with different $r$ values of surrounding tissues $(0.1,0.125$ and 0.15$)$ to evaluate the robustness of the results with respect to variation in this parameter.

Figure 5 presents the frequency-response plot of the transmitted impedance $Z t$. At high frequencies, the transmitted impedances rapidly increased because of the properties of $G_{h p}$, and they show large differences with the desired value. At low frequencies, the transmitted impedances of the conventional controller differed from the desired values. In contrast, the transmitted impedances of the integer formalization controller maintained similar values the desired one. In addition, the results also show that there is little effect of using different derivative parameters $r(=0.1$, 0.125 and 0.15 ). The frequency-response plot shows that the transmitted impedances were improved when using the proposed integer formalization controller. Telepresence is 


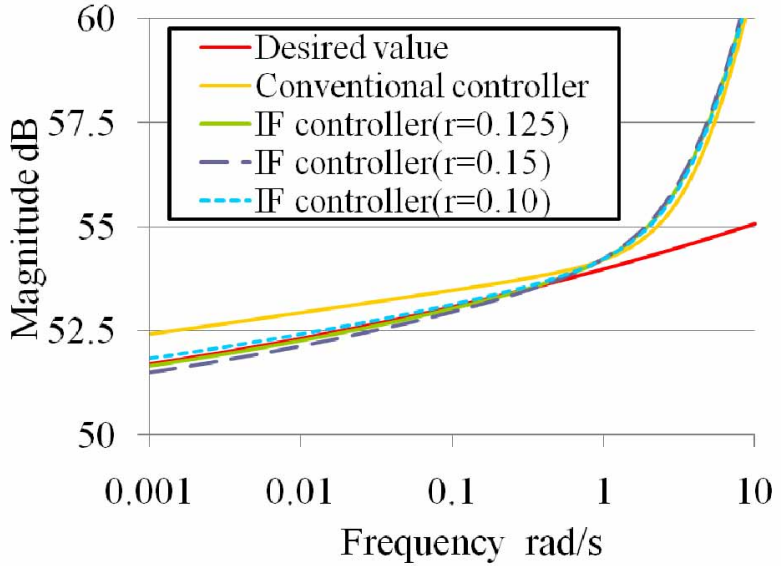

Fig. 5. Frequency response of transmitted impedance $Z_{t}$

achieved if, at low frequencies, the transmitted impedance $Z_{t}$ magnitude is within the $\pm 3 \mathrm{~dB}$ bandwidth of the desired impedance $Z_{t}$ [23]. It should be noted that the transmitted impedance $Z_{t}$ was in the $\pm 3 \mathrm{~dB}$ bandwidth of the desired one and thus telepresence was achieved across the whole frequency range.

\section{CONCLUSIONS AND Future WORK}

We investigated the performance of the controller previously proposed by several studies [13]-[16] to the case of tissue with viscoelastic properties, as modeled by a fractional derivative equation. In addition, we propose a novel controller that includes an integer formalization process suitable for viscoelastic tissue properties. The simulation results suggested that performance, in terms of force control and telepresence, became poorer when the conventional controller, which was designed for elastic target objects, was applied to viscoelastic tissues. The simulation results also suggested that our novel controller successfully improved its performance for viscoelastic tissues. The present paper only presents simulations with one degree-of-freedom robots and we suggest that experiments should be the next step in this research. An in vivo tissue experiment, for instance, with an anesthetized pig undergoing minimally invasive surgery, is one possible experimental approach.

\section{REFERENCES}

[1] R. J. Anderson and M. W. Spong. Bilateral control of teleoperators with time delay. In Proc. of the IEEE 27th Conf. on Decision and Control (CDC), Austin, Texas, USA, December 1998.

[2] H. Baier and G. Schmidt. Transparency and stability of bilateral kinesthetic teleoperation with time-delayed communication. Journal of Intelligent and Robotic Systems, 40(1):1-22, May 2004.

[3] K. H.-Zaad and S. E. Salcudean. Transparency in time-delayed systems and the effect of local force feedback for transparent teleoperation. IEEE Trans. on Robotics and Automation, 18(1):108-445, February 2002.

[4] X. Wang, P. X. Liu, B. Chebbi, D. Wang, and M. Meng. Design of bilateral teleoperators for soft environments with adaptive environmental impedance estimation. In Proc. of the IEEE Int. Conf. on Robotics and Automation, pages 1139-1144, Barcelona, Spain, April 2005.

[5] N. Hata, R. Hashimoto, and J. Tokuda. Needle guiding robot for mr-guided microwave thermotherapy of liver tumor using motorized remote-center-of-motion constraint. In Proc. of the IEEE Int. Conf. on Robotics and Automation (ICRA), pages 1664-1668, Barcelona, Spain, April 2005.

[6] G. Kronreif, M. Fürst, J. Kettenbach, M. Figl, and R. Hanel. Robotic guidance for percutaneous interventions. Journal of Advanced Robotics, 17(6):541-560, 2003.

[7] J. M. Romano, R. J. Wabster III, and A. M. Okamura. Teleoperation of steerable needles. In Proc. of the IEEE Int. Conf. on Robotics and Automation (ICRA), pages 934-939, Roma, Italy, April 2007.

[8] L. Barbe , B. Bayle, M. de Mathelin and A. Gangi, "In Vivo Model Estimation and Haptic Characterization of Needle Insertions", The International Journal of Robotics Research(2007), Vol. 26, No. 11-12, pp. 1283-1301, 2007

[9] J. Park and O. Khatib. A haptic teleoperation approach based on contact force control. The International Journal of Robotics Research, 25(5-6):575-591, May-June 2006.

[10] O. Khatib. A unified approach for motion and force control of robot manipulators: The operational space formulation. Int. J. on Robotics and Automation, 3(1):43-53, February 1987.

[11] R. Cortesão, J. Park, and O. Khatib. Real time adaptive control for haptic tele-manipulation with kalman active observer. IEEE Trans. On Robotics, 22(5):987-999, October 2006.

[12] M. Michelin, P. Poignet, and E. Dombre. Dynamic task / posture decoupling for minimally invasive surgery motions. In International Symposium on Experimental Robotics (ISER), Singapore, 2004.

[13] R. Cortesão, W. Zarrad, P. Poignet, O. Company and E. Dombre, "Haptic Control Design for Robotic-Assisted Minimally Invasive Surgery", in Proceedings of the 2006 IEEE/RSJ International Conference on Intelligent Robots and Systems, pp. 454-459, 2006

[14] W. Zarrad, P. Poignet, R. Cortesão and O. Company, "Stability and Transparency Analysis of a Haptic Feedback Controller for Medical Applications", in Proceedings of the 46th IEEE Conference on Decision and Control, pp.5767-5762, 2007

[15] W. Zarrad, P. Poignet, R. Cortesão and O. Company, "Haptic Feedback Control in Medical Robotics through Stiffness Estimation with Extended Kalman", in Proceedings of 13th International Conference on Advanced Robotics, pp.81-87, 2007

[16] W. Zarrad, P. Poignet, R. Cortesão and O. Company, "Towards Teleoperated Needle Insertion with Haptic Feedback Controller", in Proceedings of the 2007 IEEE/RSJ International Conference on Intelligent Robots and Systems, pp. 1254-1259, 2007

[17] Y. Kobayashi, J. Okamoto, M. G. Fujie, "Physical Properties of the Liver and the Development of an Intelligent Manipulator for Needle Insertion", in Proceedings of 2005 IEEE International Conference on Robotics and Automation, pp.1644-1651, 2005

[18] Y. Kobayashi, A. Onishi, H. Wtanabe, T. Hoshi, K. Kawamura, M. Hashizume and M. G. Fujie, "Development of an Integrated Needle Insertion System with Image Guidance and Deformation Simulation", in International Journal of Computerized Medical Imaging and Graphics, Vol.34(1), pp. 9-18, 2010

[19] R.L. Magin, Fractional calculus models of complex dynamics in biological tissues, Computers and Mathematics with Applications (2009), doi:10.1016/j.camwa.2009.08.039

[20] A. Lawrence. "Stability and transparency in bilateral teleoperation", IEEE Trans. on Robotics and Automation, 9(5):624-637, October, 1993.

[21] Y. Kobayashi, M. Suzuki, A. Kato, M. Hatano, K. Konishi, M. Hashizume and M. G. Fujie, "Enhanced Targeting in Breast Tissue using a Robotic Tissue Preloading-Based Needle Insertion System" in IEEE Transaction on Robotics, accepted, 2011

[22] Y. Kobayashi, T. Ando, M. Seki, T. Watanabe, M. G. Fujie, "Fractional Impedance Control for Reproducing the Material Properties of Muscle", in Proceedings of 2010 IEEE International Conference on Intelligent Robots and Systems, pp.5498-5504, 2010

[23] K. B. Fite, J. E. Speich, and M. Goldfarb. Transparency and stability robustness in two-channel bilateral telemanipulation. Journal of Dynamic Systems, Measurement, and Control, 123(3):400-407, September 2001. 